\title{
Patellar metastasis from primary tumor (Review)
}

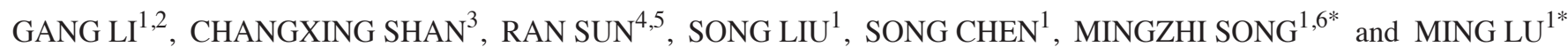 \\ ${ }^{1}$ Department of Orthopaedics, The First Affiliated Hospital of Dalian Medical University, Dalian, \\ Liaoning 116011; Departments of ${ }^{2}$ Orthopaedics and ${ }^{3}$ General Surgery, Xiangyang Central Hospital, \\ The Affiliated Hospital of Hubei University of Arts and Science, Xiangyang, Hubei 441021; \\ Departments of ${ }^{4}$ Nursing and ${ }^{5}$ Operation Room, The First Affiliated Hospital of Dalian Medical University, Dalian, \\ Liaoning 116011; ${ }^{6}$ Department of Orthopaedics, The Third Affiliated Hospital of Dalian Medical University, \\ Dalian, Liaoning 116200, P.R. China
}

Received October 23, 2016; Accepted September 1, 2017

DOI: $10.3892 / \mathrm{ol} .2017 .7478$

\begin{abstract}
Although bone tumors are frequently located in the knee area, primary tumors of the patella are rare and patellar metastases are even rarer. Knee pain is the most common complaint of patients with patellar metastases. Owing to the low incidence of patellar metastases, misdiagnosis is not unusual. The present review analyzes $\sim 44$ cases of patellar metastases originating from distinct primary sites. Reports of malignant tumors of the lung and kidney metastasizing to the patella were more common than those of other patellar metastases. Relative incidence, symptomatology, imaging features, histopathology and treatment options for these patellar metastatic lesions are described respectively along with a review of the literature. Despite numerous experiments demonstrating the reasons for implantation of tumor in patella, the answer to this question has not yet been revealed. In the light of the increasing attention on the diagnosis and the treatment of these lesions, the availability of the integrated information regarding metastases in the patella becomes more relevant.
\end{abstract}

Correspondence to: Dr Mingzhi Song or Dr Ming Lu, Department of Orthopaedics, The First Affiliated Hospital of Dalian Medical University, 222 Zhong Shan Road, Dalian, Liaoning 116011, P.R. China

E-mail: smz10gb@163.com

E-mail: luming_dmu@sina.com

*Contributed equally

Abbreviations: ESR, erythrocyte sedimentation rate; ACP, acid phosphatase; ALP, alkaline phosphatase; CEA, carcinoembryonic antigen; CRP, C-reactive protein; CT, computed tomography; MRI, magnetic resonance imaging; RCC, renal cell carcinoma

Key words: patella, patellar metastasis, symptom, diagnosis, surgery

\section{Contents}

1. Introduction

2. Carcinoma of the lung

3. Carcinoma of the kidney

4. Carcinoma of the breast

5. Carcinoma of the esophagus

6. Carcinoma of the uterine cervix

7. Carcinoma of the colon

8. Malignant melanoma

9. Other primary tumors

10. Discussion

11. Conclusion

\section{Introduction}

In the last century, primary tumors of the patella are uncommon and account for $<0.12 \%$ of bone tumors in the United States (1). The incidence of metastatic lesions to the patella is even lower (2). A Medline search from of studies published between 1960 and 2016 yielded 44 reported cases of patellar metastases. Primary tumors that have been reported to metastasize to the patella include carcinomas of the lung, kidney, breast, esophagus, uterine cervix, skin (malignant melanoma), bowel, larynx, head and neck. The reported cases of patellar metastases are presented in Table I. Of these 44 cases, $>10$ presented with anterior knee pain as the first manifestation (3-11). The mean age of the patients at presentation was 57 years (range, 37-86 years). The present study analyzed these cases of primary sites of patellar metastasis. In the present study, the metastatic patellar tumors were classified according to the primary site and type. A detailed summary and description is presented.

\section{Carcinoma of the lung}

The review of the literature revealed that metastasis from lung carcinoma is most common, with $40 \%$ of all patellar metastases originating from a primary tumor located in the lung. In total, 7 cases were associated with squamous cell 
carcinoma $(4,5,9,10,12-15)$ and 7 cases with adenocarcinoma (3,16-21). Only 1 case was adenosquamous (6), whereas for 2 cases, the histological type of lung cancer was not stated $(22,23)$. It is common for adenocarcinoma of the lung to give rise to remote metastasis prior to the onset of pulmonary symptoms, which may be attributed to the peripheral pulmonary growth of the tumor. By contrast, metastasis from lung squamous or epidermoid carcinoma is not common since these tumors are usually centrally located and therefore present with pulmonary symptoms (6).

Clinically, metastasis of the patella accompanied by knee pain was the presenting symptom of lung cancer in the patients. The common early manifestation of lung cancer is a cough and sputum production ( $75 \%$ patients), whereas bone pain as an initial symptom occurred in $22 \%$ of the patients (24). Patients who had patellar metastasis of the lung presented with progressive knee pain and limited mobility that was relieved by analgesic drugs (10). The patients frequently had a history of smoking along with a persistent cough and weight loss $(3,5,12,14,17,22)$. Additionally, 2 patients had recent trauma history $(3,9)$.

On physical examination, the diseased knee was euthermic, swollen, and tested positive in patellar shock and grinding tests $(6,10)$. A single patient exhibited inflammatory signs in the diseased knee (3). Upon palpation, the patellofemoral joint was moderately painful compared with the healthy side. Patients usually exhibited limited flexibility of the affected knee, with atrophy of the quadriceps (5). Additionally, the sounds of breathing were diminished over the pulmonary lesion when observed using auscultation $(10,12)$. Certain patients did not exhibit any symptoms of respiratory distress.

In the majority of the patients, all routine blood tests including erythrocyte sedimentation rate (ESR), C-reactive protein (CRP), acid phosphatase (ACP) and alkaline phosphatase (ALP) were within normal range (6). In a single case, the level of ESR was increased and a normochromic anemia was observed (5). Another case had an increased level of ACP (10). Serum ALP level was increased in a patient with monoarticular arthritis secondary to metastatic patellar spread of bronchogenic carcinoma (15). A Mantoux test may exclude the possibility of tuberculosis in the knee (12).

Radiographic examination of the knee commonly revealed mild osteoarthritis with a cystic lesion in the patella (Fig. 1A). The result of computed tomography (CT) imaging (Fig. 1B and C) may also indicate an extended osteolytic lesion (6). Bone scintigraphy (Fig. 1D) may reveal an increased uptake of radioisotope in the affected patella. The use of $\left[{ }^{18} \mathrm{~F}\right]$ fluorodeoxyglucose positron emission tomography/computed tomography (PET-CT) and single photon emission tomography/computed tomography were not considered to be valuable tools for the evaluation of the malignant tumors $(13,16)$. However, the PET-CT showed a hypercaptation of the pulmonary lesions, of a conglomerate lymph node mass in the hilum and of the patellar lesion, suggestive of active metastatic disease. Arthroscopy was helpful to provide insight into the intra-articular situation including synovial effusion and general degenerative changes. On occasion, soft blue cartilage was indicative of the pathological change. The radiographs of the chest, bronchoscope and thoracic multi-slice computed tomography were necessary for diagnosis of the primary lesion, since it was possible to easily identify a mass in the lobe $(6,13,22,25)$. Excision biopsy was performed and the diagnosis was confirmed using pathological testing. Immunohistochemically, the ceroplastic cells co-expressed keratin and vimentin. Carcinoembryonic antigen (CEA) and epithelial membrane antigen (EMA) were also present in certain cases. Thyroid transcription factor-1 (TTF-1) confirmed the nuclear positivity in the majority of adenocarcinoma cells, which was suggestive of the primary lung process. However, the squamous cells were TTF-1-negative and cytokeratin 5/6-positive which confirmed the adenosquamous nature of the lung carcinoma (6).

For patellar malignant tumors, surgery was the optimal therapeutic option. The treatment of patellar metastasis is dependent on the size of the lesion. Early deposits without soft-tissue extension may be treated by patellectomy and reconstruction of the extensor mechanism $(6,13,16)$. Postoperatively, the patients wore a knee immobilizer (9). Nevertheless, larger lesions with soft-tissue extension required a wide excision, followed by systemic chemotherapy and local radiation therapy (16). In one case, the size of the lesion was $>50 \%$ of the patella and the anterior cortex was not intact. Aktas et al (10) adopted total patellectomy and total synovial resection for diagnostic and therapeutic purposes. This may help patients to improve their quality of life by eliminating severe pain and disability (10). The majority of the patients succumbed to progressive lung disease within a short postoperative period $(3,9,10,14,15,17)$. However, 2 patients presented asymptomatically and were capable of full functioning of the knee with no recurrence $(6,13)$.

\section{Carcinoma of the kidney}

The cases of metastatic tumor from renal cell carcinoma (RCC) account for $14 \%$ of all patellar metastases. Howlett and Caranasos (25) described a patient with an arteriovenous shunt secondary to metastatic patellar spread of renal carcinoma.

Clinically, $30 \%$ of patients with RCC present with symptoms. It is associated with metastatic disease. The classic symptomatic triad of hematuria, loin pain and loin mass is uncommon (10\%) and only observed in advanced cases (26). Long-term knee pain was the main complaint of the patients, who were often no longer able to walk or move. There was only one case with associated trauma (8).

Additionally, 1 patient presented with knee pain due to a pathological fracture (27). A physical examination of the affected knee revealed swelling, tenderness, mild erythema and painful flexion-extension motion $(7,8,27)$. No other notable physical results were observed.

Laboratory investigations including a full blood count, routine biochemistry, ESR, CRP and prostate-specific antigen (PSA) levels did not reveal any notable abnormalities (8).

$\mathrm{X}$-ray (Fig. 2A) revealed a lucency within the patella and a discontinuity in the cortex of the patella with an osteolytic lesion $(7,8,11,27)$. CT imaging (Fig. 2B and C) identified a large lucent area occupying the majority of the patella with multiple areas of cortical disruption along its border $(7,8)$. A radioisotope bone scan (Fig. 2D) revealed isolated abnormal tracer activity local to the patella (7). A renal ultrasound revealed a large mass arising from the superior pole of the kidney (7). Subsequent CT scanning of the chest, abdomen 


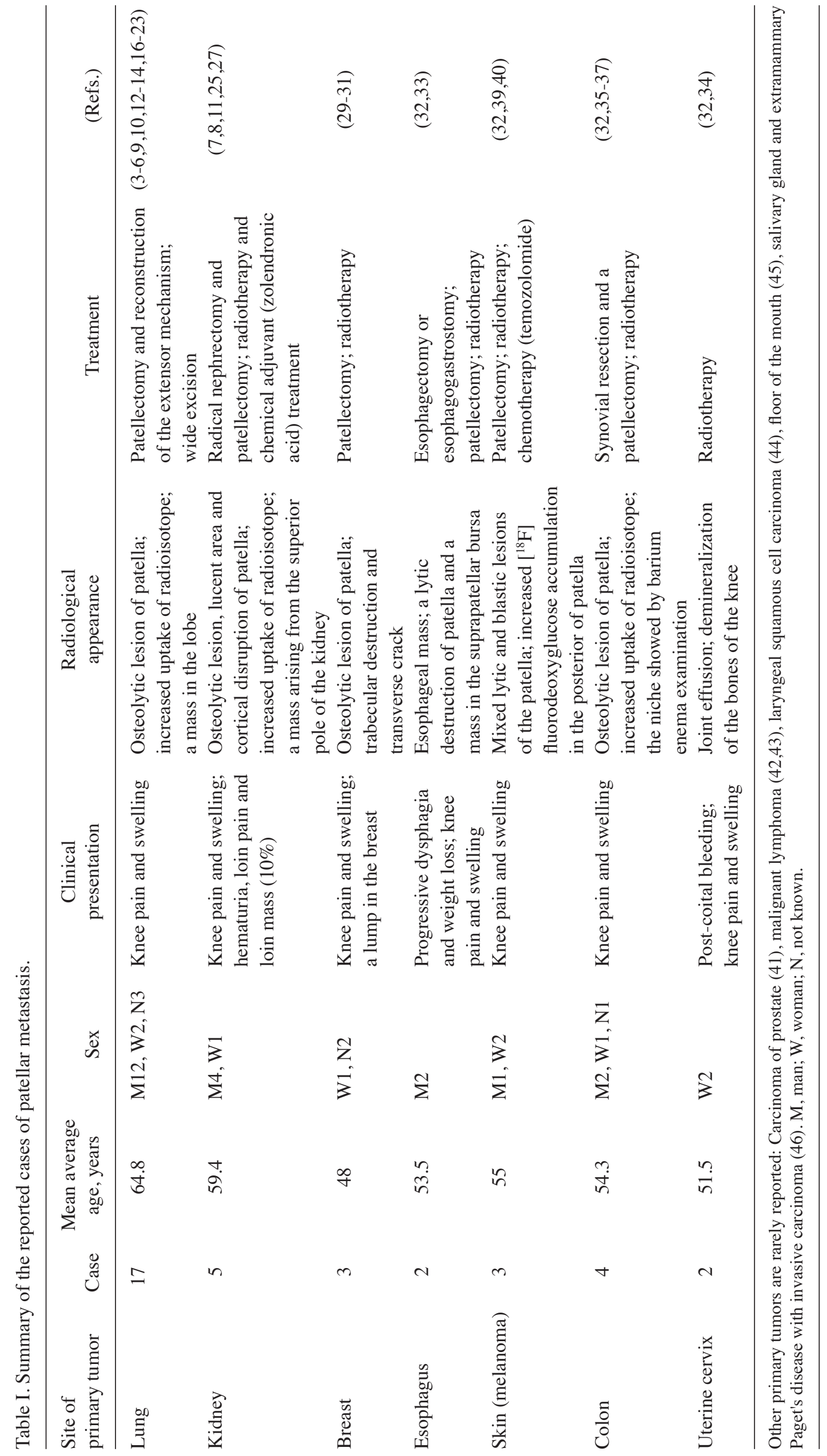


and pelvis contributed to the diagnosis of the primary lesion and identified the stage of disease; a mass was easily located in the pole of the kidney. The appearance of the mass was consistent with a primary RCC. Histological examination of the resected patella indicated metastatic renal adenocarcinoma.

Surgery remains the main method of treatment. A radical nephrectomy and patellectomy were performed, which may be crucial as there was no evidence of other metastases, although the presentation as a pathological fracture may make dissemination easier (7,8,27). Lim et al (8) adopted cryotherapy to realize a unique anatomical preservation on the basis of two principles. First, the treatment is suitable for the patient with a short life expectancy on the basis of the American Joint Committee on Cancer (AJCC) Staging for stage 4 RCC. Secondly, RCC metastasis presents a unique histological type of bony metastasis in which disease control concepts appear to be similar to primary bone disease, with no survival rate advantage achieved by performing a wide resection as opposed to intralesional curettage and local stabilization $(8,28)$. In addition, radiotherapy and chemical adjuvant (zolendronic acid) treatment for the patellar metastasis were reported in several cases (7). Postoperatively, the patients exhibited no evidence of progressive disease and were capable of full motion of the affected knee $(8,11)$. Additionally, 2 patients accepted a conservative treatment that was not well-described in the report. There was no further information regarding follow-up $(7,25)$.

\section{Carcinoma of the breast}

Patellar metastases originating from breast tumors are uncommon with an incidence of $2 \%$ among all patellar metastases.

Clinically, a lump or a change in the shape of the breast may be the first observable manifestation in the patients $(29,30)$. Specific cases may exhibit progressive knee pain, swelling and erythema (31). However, none of the patients had a history of knee trauma or signs of inflammation.

Upon physical examination, a change in the size or shape of the breast may be observed and a lump in the breast felt (29). The patient may exhibit swelling, tenderness, moderate effusion and decreased range of motion in the affected knee (31). The majority of routine laboratory findings were within normal ranges.

Radiographs (Fig. 3A) demonstrated osteolytic deposits and an area of trabecular destruction of the patella (31). Mammogram, ultrasound and bone scintigraphy were meaningful to the determination of the patellar metastasis.

Histological examination revealed that the spheroidal cells replacing the trabecular bone of the patella and filling the center of the field with normal bone on either side were similar to those in the primary breast carcinoma.

A patellectomy was performed followed by routine physiotherapy. Radiotherapy was used to target the internal mammary and mediastinum, with Prednisone supplemented. The knee pain was relieved and effusion was gradually aspirated. The general condition of 1 case deteriorated rapidly and the patient succumbed following a short period of time (31).
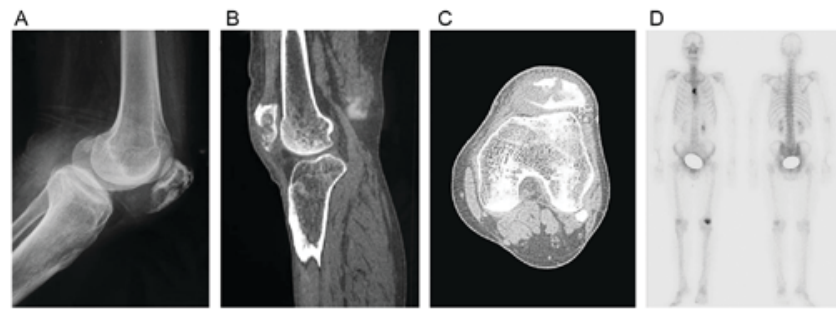

Figure 1. The imaging features of patellar metastases from lung carcinoma. (A) Radiograph and (B) computed tomography (CT) scan on the left patella revealed an osteolytic lesion. (C) Axial CT identified soft tissue swelling surrounded the lesion. Additionally, a [ $\left.{ }^{99 \mathrm{~m}} \mathrm{Tc}\right]$ methylene diphosphate bone scintigraphy (D) indicated increased radioactivity in the pulmonary hilum and the left patella.
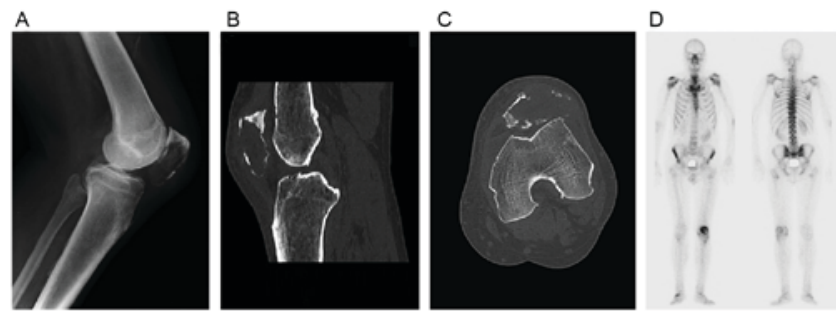

Figure 2. The imaging features of patellar metastases from renal carcinoma. (A) Lateral radiograph and a (B) sagittal and (C) transverse computed tomography scan of the affected patella indicated a large lucency within the patella and a discontinuity in the anterior cortex of the inferior pole suggesting pathological fracture. (D) A bone scan demonstrated an isolated abnormal tracer activity local to the left patella.
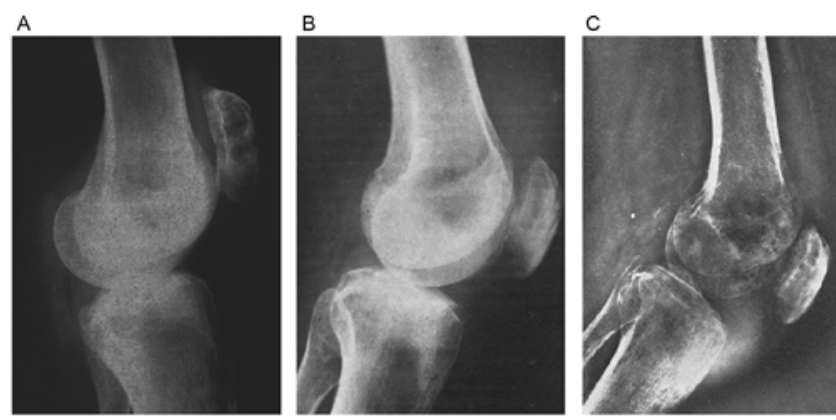

Figure 3. Radiography of other three patellar metastases. Patellar metastases from (A) breast cancer, (B) esophageal carcinoma and (C) uterine cervix carcinoma all revealed demineralization and lytic destruction of the affected knee.

\section{Carcinoma of the esophagus}

Metastasizing from the esophagus is rare, with an incidence of $5 \%$ of all patellar metastases.

Normally, the patients initially had a history of progressive dysphagia and weight loss. Immobilizing pain, swelling and effusion in the affected knee developed gradually $(32,33)$.

Physical examination revealed considerable swelling, warmth and knee pain $(32,33)$. Patients typically had a limited range motion of the affected knee. Certain patients did not seek medical attention until pain and disability became deteriorative (33). Results of routine laboratory studies, including blood cell count, ESR, blood calcium and ALP, were demonstrated to be normal (32). 
Radiographs (Fig. 3B) of the knee presented a lytic lesion, demineralization of the patella and a mass in the suprapatellar bursa. An arthrogram and arteriography demonstrates the marked vascularity in the areas of irregular synovium which contained the metastatic nodules $(32,33)$. An arthrocentesis collected synovial fluid contaminated with blood, which yielded Staphylococcus aureus in culture. A barium-swallow examination and esophagoscopy revealed an esophageal mass. Following the examination of a tissue biopsy, the mass was revealed to be an epidermoid carcinoma (33). Stoler and Staple (32) reported that a closed patellar biopsy, two Cope needle biopsies of the synovium and cytology of the joint fluid may be used for the diagnosis. However, an open patellar biopsy or arthrotomy may be recommended when the results were negative $(32,33)$.

Metastatic epidermoid carcinoma consistent with an esophageal origin was confirmed in permanent pathological sections.

The optimal treatment approach remains unknown. Ashby and Dapper (33) promoted a surgical approach. In 1 case, an esophagectomy and esophagogastrostomy were performed following a total patellectomy. In the postoperative period, radiation therapy was valuable in the relief of pain. However, Stoler and Staple (32) indicated a conservative treatment that was not well-described in the report. The 2 cases had no follow-up.

\section{Carcinoma of the uterine cervix}

Metastasizing from uterine cervix is rare $(\sim 2 \%$ of all patellar metastases), and only a small number of cases of this type was reported.

Generally, the patients presented with post-coital bleeding (32). The pain, swelling and progressive disability of the affected knee may often disturb the patients $(32,34)$.

Upon physical examination, symptoms including warmth, swelling and tenderness of the patella were observed. There was redness and a localized increase in temperature. The range of motion was limited. Occasionally, clinical examination could not be entirely completed due to severe pain (34). No other joints were affected.

Routine laboratory studies and ESR of reported cases were normal (32). Radiographs of the diseased knee (Fig. 3C) revealed minimal joint effusion, lytic destruction and demineralization of the bones of the knee (32). An open biopsy of the synovium and patella determined metastatic epidermoid carcinoma. Stoler et al (32) reported that radiation therapy is the treatment of choice and it provides significant symptomatic relief.

\section{Carcinoma of the colon}

Metastasizing from colon is rare, with an incidence of $~ 9 \%$ of all patellar metastases.

Clinically, the patients always presented with increasing knee pain accompanied by swelling $(32,35-37)$, which persistently affected mobility. Furthermore, 1 case was associated with traumatic history and aggravated by motion (36).

Upon physical examination, warmth, tenderness, swelling and limited extension of the diseased knee were observed $(32,36,37)$. No other joints were involved and examination of the other knee was not remarkable. Routine laboratory studies revealed that ESR and CEA levels were within normal ranges $(35,36)$.

Radiographs revealed osteolytic lesions and destruction of the subchondral bone of the patella. Further radiography studies may demonstrate a lytic lesion in the skull or a mass in the lung. Bone scintigraphy revealed dense hyperfixation of the affected patella (36). Barium enema examination revealed a lesion in the midsigmoid colon suggesting the presence of a tumor (32). Arthrotomy was performed and revealed that the patella was markedly friable, although the synovium was normal. Pathological examination of the biopsy, stained with hematoxylin-eosin-safran, revealed the diagnosis of patellar metastasis from a well-differentiated large bowel adenocarcinoma (36).

Urvoy et al (36) were in favor of surgical treatment. A partial colectomy was performed followed by a total patellectomy and extensive synovial resection according to the technique of Boyd and Hawkin (38). Following surgery, the patient was asymptomatic and capable of full functioning of the affected knee. Postoperative radiotherapy was administered to complement the surgical treatment (36). However, Stoler and Staple (32) reported that the patient was treated only with radiotherapy and discharged. The treatment of the other cases was not well-described in the report.

\section{Malignant melanoma}

The tumors originating from malignant melanoma account for $\sim 7 \%$ of all patellar metastases. Melanoma is well-known for its capacity to metastasize to numerous sites, including certain places rarely observed with other solid tumors.

Clinically, the patients presented with increasing anterior knee pain and swelling in the affected knee $(32,39,40)$. Patients were not able to bear weight on the affected leg for more than a short period of time. Only 1 case had a relevant medical history of a melanoma on the back and underwent wide excision of lesion followed by lymphadenectomy (40).

Upon physical examination, symptoms including mild pain, mass, swelling, tenderness, heat and limited motion of the patellar were observed $(32,39,40)$. Furthermore, the quadriceps muscle was atrophied (39). In 2 cases, multiple metastases were established in lymph nodes of different regions (32).

Routine laboratory studies revealed that ESR and other blood tests were within normal ranges. However, 1 case revealed an increase in the white blood cell count and plasma (39).

A radiograph of the affected knee demonstrated mixed lytic and blastic lesions of the patella. The cortex was intact without periosteal reaction (39). A chest X-ray revealed large basal opacities suggestive of multiple metastases (39). PET-CT was performed to investigate metastatic areas. Increased $\left[{ }^{18} \mathrm{~F}\right]$ fluorodeoxyglucose accumulation in the patella was observed with an abnormal standardized uptake rate (40).

Histopathological examination of the skin biopsy revealed a superficial spreading type of malignant melanoma with an invasion; the changes in the patella confirmed metastatic malignant melanoma (39).

Treatment options for the tumor originating from malignant melanoma remain in dispute. Jaeger et al (39) were in favor of a surgical approach. A patellectomy was performed on 
a patient and the pain in the affected knee was relieved. In the postoperative period, the condition of the patient deteriorated with weight loss, diarrhea and generalized abdominal tenderness. The patient succumbed following a short period of time. Tas and Keskin (40) reported that the patient was treated with single-agent temozolomide chemotherapy. In another case, the patient was treated conservatively and discharged (32). The 2 cases had no follow-up.

\section{Other primary tumors}

Other primary tumors metastasizing to the patella were also reported, including carcinoma of prostate (41), malignant lymphoma $(42,43)$, laryngeal squamous cell carcinoma (44), floor of the mouth (45), salivary gland and extramammary Paget's disease with invasive carcinoma (46).

\section{Discussion}

Metastasis of any type to the patella is rare in comparison with primary tumors of the patella. Case reports and the associated review articles of the primary patellar tumors may be easily searched (47). Nevertheless, only a small number of reports related to patellar metastasis has been published in the scientific literature.

The patella stems from a cartilaginous precursor in the third month of gestation and ossifies at $\sim 3$ years of age. Its ossification is similar to that of an epiphysis or apophysis of a long bone. This makes the patella a possible site of bony lesions (2). The patella is a sesamoid bone with a relatively poor vascular supply, consisting of a number of nutrient branches from the collateral vessels of the knee. Thus, patellar circulation may not be sufficient for tumor cells to occur and metastasize (48). Furthermore, the small size of the patella and its dense cancellous structure make the bone an unlikely site for a metastatic deposit. Sosnoski et al (49) revealed that trauma may be a factor in determining the establishment of a circulating tumor embolus. Although the theory illustrating patellar metastases has reached an agreement in the majority of the literature, there remains another hypothesis. Lim et al (8) reported that the tumor emboli did not exhibit preferential metastasis to highly vascularized bones including the lumbar spine. This raises the consideration of tissue tropism as another aspect influencing carcinogenesis (50). A previous study revealed that the probability of disease-free and overall survival rates in colorectal cancer was dependent on lymph node metastasis and the degree of tumor differentiation, but not on the presence of circulating tumor cells (51). Tissue tropism has previously become a focus as another important factor in tumor metastases. Kang (52) demonstrated the development of mutations allowing metastasis in a targeted tissue-specific manner in one experiment. The author insisted that it is possible that the patient may possess a molecular make-up of the primary tumor enabling preferential metastasis to the patella. Therefore, further molecular studies are required in order to determine the tumor tropism of the primary deposits.

In the review of the literature, there were $\sim 20$ patients with patellar metastasis (lung, kidney, breast, esophagus, uterine cervix, colon and skin) in the left knee compared with 9 cases in the right side. For potential patients suffering from left patellar tumors, more attention should be paid to the possible diagnosis of patellar metastasis.

The most common symptom of patellar metastases is anterior knee pain that usually lasts a marked period of time from pain onset to determination via diagnosis. In certain cases, it is the only sign of the patellar metastases. Certain patients complain of immediate patellar pain following unimpressive injury that may be the result of a pathological fracture. Swelling is the second most common symptom and occurs in multiple cases of patellar tumors. The impaired function of flexion and extension upon physical examination is easier to determine. Affected by pain and swelling, the range of motion of the diseased knee is decreased. With the limitation of motion and limp, patients may experience muscular atrophy of the quadriceps. Additionally, tumor mass is another symptom which is often revealed through palpation during a physical examination. It is not only primary malignancies but also aggressive tumors of the patella which are able to raise local redness, heat, effusion and other inflammatory responses. Furthermore, it is hypothesized that there may be an association between the lesion site and the tumor type. However, with asymptomatic knee lesions, patients may present with the typical symptoms of the primary tumor site, including cough and sputum of lung tumors, progressive dysphagia and weight loss of esophageal tumor and breast lump of the breast tumor. When signs of weight loss, night sweats and other cachexia appear, the possibility of other malignant tumors should be considered.

However, more evidence is required to distinguish patellar metastases from primary tumors and other types of lesion. In a clinical setting, a painful swollen knee is commonly associated with septic arthritis, rheumatic arthritis and degenerative osteoarthritis. Certain authors described knee symptoms as marked. Although a tumor is not a frequently suspected cause of anterior knee pain, the possibility of patella metastasis should not be ignored in patients with malignancy. As the patellar neoplasm is such a rare etiology as anterior knee pain, the determination is often postponed.

In patients suffering anterior knee pain, a radiograph is the most common method to identify patellar anomalies and may contribute to the early diagnosis of a patellar tumor (53). The traits of the X-ray including bone destruction, extent, margins, cortex, sclerotic rim, calcifications, ossification, periosteal reaction, pathological fracture and surrounding involvement should be considered carefully. Malignant tumors are frequently identified with periosteal reaction, permeative destruction, total patellar extension and ossifications or pathological fracture. Occasionally, radiographic diagnosis is insufficient due to metastatic cancer, Paget's disease, plasmacy toma, osteomyelitis, tuberculosis hyperparathyroidism, gout and other lytic neoplastic lesions of the patella. Compared with plain radiograph, bone scintigraphy is more useful in the early diagnosis of bone metastasis. Primary patellar tumors are often not studied through imaging results, which are otherwise helpful to determine the stage of the lesion and the appropriate surgical course. The combination of X-ray, CT, MRI and PET-CT is particularly necessary for determination of diagnosis, involvement of the surrounding tissue and precise tumor location. Furthermore, a bone scan appears to 
lack the sensitivity required to indicate patellar tumors. Only a small number of the cases were identified with an increased focal activity on the lesion. In addition, according to the primary symptoms, chest radiograph, abdominal $\mathrm{CT}$, renal ultrasound or barium enema examination is recommended for all the patients with patellar tumors. This is meaningful for determining diagnosis as well as guiding treatment plan.

Owing to the rarity of reports and unusual changes, the reported reviews of laboratory tests on the patellar tumors remain limited. With the increase in relative cases, a certain amount of data has been accessible. Although the routine laboratory tests are usually within the normal ranges, an increase in levels of ESR, serum calcium, ACP or ALP may be detected in certain malignant cases. A known tumor marker, CEA, is beneficial for diagnosis of the malignant tumor. Examination of the synovial fluid has also been utilized as a diagnostic technique, which is of marked importance in certain cases. Thus, it is important that clinicians do not overlook the results of laboratory tests. Radiographic features of primary patellar tumors are atypical of a specific histotype. Histological examination remains the gold standard diagnostic technique; however, in the light of the risk of contamination, a needle biopsy is considered a reasonable alternative. The incisional biopsy is recommended only if the diagnosis and the radiographic features are paradoxical. Two key points should be emphasized: i) During a needle biopsy, the joint must not be entered; ii) during an incisional biopsy, the patella and quadriceps tendon, the joint cavity and the synovial membrane must not be contaminated (2). Applying cytological techniques, identification of cells should raise the attention of physicians, particularly when differentiating diagnosis from inflammatory joint disease. Joint aspiration and needle biopsy should first be attempted prior to open biopsy. However, only the histological examination of the specimen is a gold standard to indicate the origin of the tumor.

The treatment approaches for patellar metastasis include surgery, radiotherapy and chemotherapy. Surgery is the optimal method for the majority of symptomatic patients who possess a patellar tumor. Furthermore, according to distinct symptoms of patients, suitable surgical options may include simple curettage with bone grafting, excision, patellectomy, extensor mechanism reconstruction and knee resection to above-knee amputation. In order to avoid tumor spreading in the joint, extreme care should be taken when curettage is performed. If the peripheral shell of reactive bone cannot be retained according to preoperative radiographic results, it is preferable to excise the patella. In order to preserve motional function, the intact extensor apparatus should be maintained or extensor structure be reconstructed. In the place of patellectomy, cryotherapy allows anatomical preservation. The patella-preserving surgery may achieve local remission and preserves the patient's quality of life (8). The appropriate surgical intervention combined with radiotherapy and chemotherapy may be rational due to regional invasion or metastases. Radiotherapy has been used in patellar metastases following patellectomy and extensive resection of soft tissue. For certain postoperative tumors, radiotherapy was deemed inappropriate as a result of radiation-induced malignant degeneration (54). Radiotherapy was only utilized in multiple malignant lesions, particularly hematological and vascular malignancies (2). Neoadjuvant chemotherapy has become a potential treatment for specific patellar malignant tumors. Of course, attention should also be paid to the treatment of the primary malignancies. Usually, combined therapy of multiple sites is necessary.

\section{Conclusion}

These cases were summarized in order to highlight a number of simple yet powerful learning points: Despite being rare, during the differential diagnosis of a common orthopedic symptom, anterior knee pain, one should be aware of the rare possibility that it could be caused by patellar metastasis. More unidentified cases of patellar metastasis may be gradually confirmed. Limited by the knowledge of the characteristics of patellar metastasis, the integrated diagnosis should be considered seriously. The extended application of CT, MRI and even PET-CT is necessary. Innovative non-invasive biopsy is required to aid specimen collection without resulting in contamination. With improved oncological management of patients, the functional outcome of treatment may be considered, resulting in less debilitating surgery. On occasion, in order to relieve the anterior knee pain and retain patient function, the more standard and effective treatment ought to be outlined individually. With an increasing focus on the diagnosis and treatment of these lesions, further types of patellar metastasis are expected to be reported in the future.

\section{References}

1. Unni KK: Osteosarcoma. In: Dahlin's bone tumors. General aspects and data on 11.087 cases. Devaney, K (ed). 5th edition. Lippincott-Raven Publishers, Philadelphia, pp143-184, 1996.

2. Mercuri M and Casadei R: Patellar tumors. Clin Orthop Relat Res 389: 35-46, 2001.

3. Sousa M, Melo V, Silva E and Vale J: Patellar metastasis as the first manifestation of an adenocarcinoma of the lung. Chest 145: 316A, 2014

4. Feng H, Li H, Wang J, Zhang X and Feng J: Squamous carcinoma of the lung metastases to the patella. Clin Nucl Med 40: 504-505, 2015.

5. Pazzaglia UE, Barbieri D and Cherubino P: Solitary metastasis of the patella as the first manifestation of lung cancer. Int Orthop 13: 75-76, 1989.

6. Tudor A, Sestan B, Jonjic N, Miletic D, Hadzisejdic I, Prpic T and Rakovac I: Solitary metastasis of the patella in the differential diagnosis of anterior knee pain. West Indian Med J 59: 110-112, 2010.

7. Broomfield J, Ralte P, Morapudi S, Vasireddy N and Kershaw S: Anterior knee pain: An unusual presentation of renal cell carcinoma. J Surg Case Rep 2014: rju018, 2014.

8. Lim CT, Wong AS, Chuah BY, Putti TC, Stanley AJ and Nathan SS: The patella as an unusual site of renal cell carcinoma metastasis. Singapore Med J 48: e314-e319, 2007.

9. Sun EC, Nelson SD, Seeger LL, Lane JM and Eckardt JJ: Patellar metastasis from a squamous carcinoma of the lung: A case report. Clin Orthop Relat Res 391: 234-238, 2001.

10. Aktas S, Demiral H, Bilgi S, Caglar T and Calpur OU: Patellar metastasis from a lung epidermoid carcinoma. Yonsei Med J 39: 474-177, 1998.

11. Kwa S and Nade S: Metastasis in a patella: A rare site. Aust N Z J Surg 59: 351-352, 1989.

12. Sur RK, Singh DP, Dhillon MS, Gupta BD, Murali B and Sidhu R: Patellar metastasis: A rare presentation. Br J Radiol 65: 722-724, 1992.

13. Wu B, Xiu Y, Jiang L and Shi H: SPECT/CT imaging of patella metastasis from a squamous carcinoma of the lung. Clin Nucl Med 38: 125-127, 2013. 
14. Benedek TG: Lysis of the patella due to metastatic carcinoma. Arthritis Rheum 8: 560-567, 1965.

15. Gall EP, Didizian NA and Park Y: Acute monarticular arthritis following patellar metastasis, a manifestation of carcinoma of the lung. Jama 229: 188-189, 1974.

16. Codreanu I, Zhuang H, Alavi A and Torigian DA: Patellar metastasis from lung adenocarcinoma revealed by FDG PET/CT. Clin Nucl Med 37: 623-624, 2012.

17. Ganjoo KN, Loyal JA, Cramer HM and Loehrer PJ: Lung cancer presenting with solitary bone metastases. Case 1: Metastatic adenocarcinoma to the patella. J Clin Oncol 17: 2995-2997, 1999.

18. Pauzner R, Istomin V, Segal-Lieberman G, Matetzky S and Farfel Z: Bilateral patellar metastases as the clinical presentation of bronchogenic adenocarcinoma. J Rheumatol 23: 939-941, 1996.

19. Cooper ME and Mess D: Isolated skeletal metastasis to the patella. Am J Orthop (Belle Mead NJ) 29: 210-212, 2000.

20. Rosenthal MA and Tiver KW: Patellar metastases in the presence of chondrocal cinosis. Australas Radiol 35: 197-198, 1991.

21. Patel MR and Desai SS: Patellar metastases. A case report and review of the literature. Orthop Rev 17: 687-690, 1988.

22. Humphreys L and Sridhar M: Patellar metastasis. Lancet 359: 1739,2002

23. Meddeb N, Hamza S, Moalla M, Siala M and Sellami S: Patelar metastasis of primary lung cancer. Rev Pneumol Clin 59: 176-178, 2003 (In French).

24. Hyde L and Hyde CI: Clinical manifestations of lung cancer. Chest 65: 299-306, 1974.

25. Howlett SA and Caranasos GJ: Metastatic renal cell carcinoma producing arteriovenous shunt. Arch Intern Med 125: 493-495, 1970.

26. Doshi D, Saab M and Singh N: Atypical presentation of renal cell carcinoma: A case report. J Med Case Rep 1: 26, 2007.

27. Warner GC, Dodds RDD and Malone PR: Pathological fracture of the patella as the first presentation of renal cell carcinoma. Knee 6: 281-283, 1999.

28. Nathan SS, Lim CT, Chuah BY, Putti TC, Stanley AJ and Wong AS: Renal cell carcinoma bony metastasis treatment. Ann Acad Med Singapore 37: 247-248, 2008.

29. Keeley CD: Bilateral patellar metastases from carcinoma of the male breast. Can J Surg 16: 328-329, 1973.

30. Taylor GH: Pathologic fracture of the patella caused by metastatic carcinoma. N Y State J Med 64: 430-431, 1964.

31. Klenerman L: A Metastatic deposit in the patella from a carcinoma of the breast. Postgrad Med J 41: 284-286, 1965.

32. Stoler B and Staple TW: Metastases to the patella. Radiology 93 853-856, 1969.

33. Ashby ME and Dappen N: Esophageal carcinoma metastatic to the patella. A case report. Jama 235: 2519-2520, 1976.

34. Tos L and Salvi V: Tumori Della Rotula. In Tos L, Salvi V (eds) La Patologia Non Traumatica Della Rotula. Torino, Edizioni Minerva Medica 195-208, 1968.
35. Griener B and Müller-Färber J: Patellar metastasis of colon carcinoma. Rare occurrence in differential diagnosis of acute knee pain: Case report. Unfallchirurg 104: 778-781, 2001 (In German).

36. Urvoy P, Mestdagh H, Butin E, Lecomte-Houcke M and Maynou C: Patellar metastasis from a large bowel adenocarcinoma. Acta Orthop Belg 59: 409-411, 1993.

37. Weinert CR and Wiss DA: Unusual lesions of the patella. Orthopedics 2: 378-383, 1979.

38. Boyd HB and Hawkins BL: Patellectomy; a simplified technique. Surg Gynecol Obstet 86: 357, 1948.

39. Jaeger HJ, Kruegener GH and Donovan AG: Patellar metastasis from a malignant melanoma. Int Orthop 16: 282-284, 1992.

40. Tas $\mathrm{F}$ and Keskin S: Patellar metastasis of melanoma. Indian J Med Res 138: 370, 2013.

41. Cole WH: Primary tumors of the patella. J Bone Joint Surg 23: 637-654, 1925

42. Ehara S, Khurana JS, Kattapuram SV, el-Khoury GY and Rosenthal DI: Osteolytic lesions of the patella. AJR Am J Roentgenol 153: 103-106, 1989.

43. Sutro CJ: Lymphosarcoma of the patella: Radical excision without repair of the extensor apparatus of the leg. Bull Hosp Joint Dis 24: 68-74, 1963.

44. Choi YS, Yoon YK, Kwak HY and Song IS: Patellar metastasis from squamous cell carcinoma of the larynx. AJR Am J Roentgenol 174: 1794-1795, 2000.

45. Singh HK, Silverman JF, Ballance WA jr and Park HK: Unusual small bone metastases from epithelial malignancies: Diagnosis by fine-needle aspiration cytology with histologic confirmation. Diagn Cytopathol 13: 192-625, 1995

46. Kawamura H, Ogata K, Miura H and Sugioka Y: Patellar metastases: A report of two cases. Int Orthop 17: 57-59, 1993.

47. Song M, Zhang Z, Wu Y, Ma K and Lu M: Primary tumors of the patella: World J Surg Oncol 13: 163, 2015.

48. Scapinelli R: Blood supply of the human patella. Its relation to ischaemic necrosis after fracture. J Bone Joint Surg Br 49: 563-570, 1967.

49. Sosnoski DM, Norgard RJ, Grove CD, Foster SJ and Mastro AM: Dormancy and growth of metastatic breast cancer cells in a bone-like microenvironment. Clin Exp Metastasis 32: 335-344, 2015.

50. Fearon ER and Vogelstein B: A genetic model for colorectal tumorigenesis. Cell 61: 759-767, 1990.

51. Bessa X, Elizalde JI, Boix L, Piñol V, Lacy AM, Saló J, Piqué JM and Castells A: Lack of prognostic influence of circulating tumor cells in peripheral blood of patients with colorectal cancer. Gastroenterology 120: 1084-1092, 2001.

52. Kang Y: Breast cancer bone metastasis: Molecular basis of tissue tropism. J Musculoskelet Neuronal Interact 4: 379-380, 2004

53. Ferguson PC, Griffin AM and Bell RS: Primary patellar tumors. Clin Orthop Relat Res 336: 199-204, 1997.

54. Okada K, Sato K, Abe E, Kataoka Y, Miyakoshi N, Ishikawa N and Sageshima M: Case report 858: Postradiation osteosarcoma of the patella. Skeletal Radiol 23: 471-474, 1994. 